\title{
THE ORIGIN OF THE ASTEROID RING
}

S. GÅs K A

Astronomical Institute, N. Copernicus University, Toruń, Poland

\begin{abstract}
From extended statistical investigations of the orbital elements of asteroids and of their dispersions it is found that the relations for some of the dispersions are stable and are regarded to be invariable. By assuming that the orbital elements derived by putting the dispersions equal to zero correspond to those of the mother planet of the asteroid ring, it is found that the mother planet is Mars.
\end{abstract}

In this paper statistical investigations are made for the orbital elements of asteroids and their dispersions. The data used are osculating elements tabulated in the Ephemerides of Minor Planets for 1969 published by the Institute of Theoretical Astronomy at Leningrad, secular elements published by Brouwer (1950) and Jacobi's constant published by Sultanov and Ibragimov (1962).

It is well-known that the distribution function $n(\Pi)$ shown by dots in Figure 1, where $n$ is the number and $\Pi$ is the longitude of the perihelion, has the maximum at $\Pi \cong 10^{\circ}$ and the minimum at $\Pi \cong 180^{\circ}$. The maximum is especially prominent for $e>0.14$ (Gąska, 1970). The distribution of points in $(\Pi, \varphi)$-plane $(e=\sin \varphi)$ suggests a strong correlation between $\Pi$ and $\varphi$. The mean values of $\varphi$ taken for arbitrary intervals of $\Pi$ are shown as circles in Figure 1, which indicates that the relation $\bar{\varphi}(\Pi)$ can be represented by two straight lines. Similar results are derived even if the asteroids; for which the longitude of the perihelion is of libration with respect to those of Jupiter or Jupiter and Saturn, are rejected.

Similar statistics are performed for the two secular elements, the proper eccentricity, $A$, and the proper longitude of the perihelion, $\Pi_{1}$. In Figure 2 the distribution

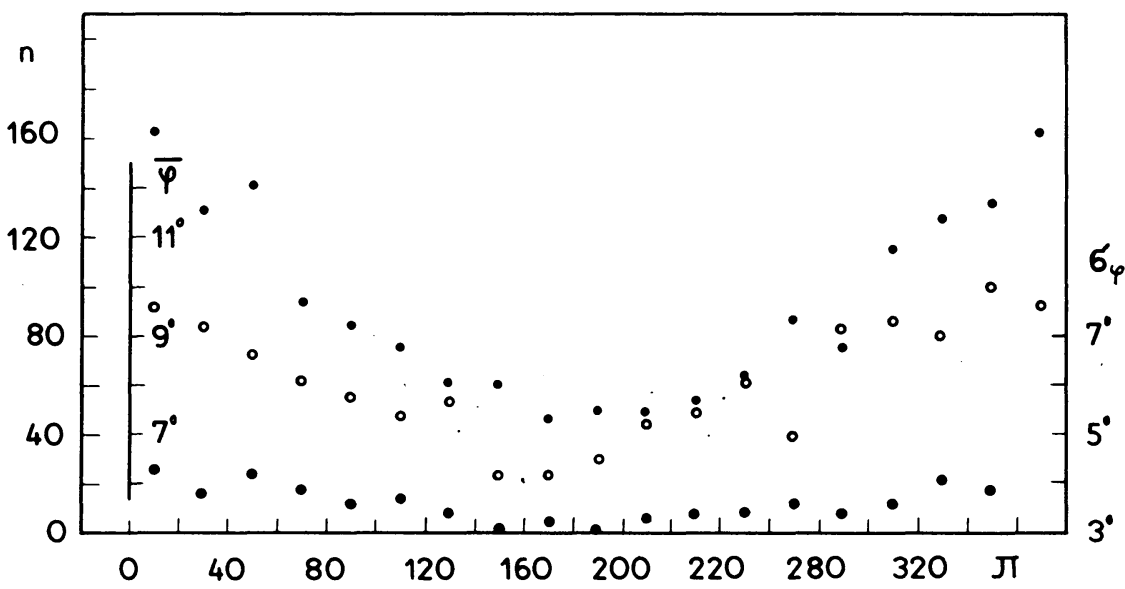

Fig. 1. The distribution function $n(\Pi)$ (dots), the mean value of $\varphi$ (open circles), and the dispersions of $\varphi$ (black circles) vs $\Pi$ are shown. 
function $n\left(\Pi_{1}\right)$, mean values of $A$, and the dispersions of $A$ are shown as functions of $\Pi_{1}$ by dots, open circles, and black circles, respectively.

Comparing Figure 1 with Figure 2 we come to the following conclusions: (1) The distribution functions for $\Pi$ and $\Pi_{1}$ are almost identical. (2) The dispersions are

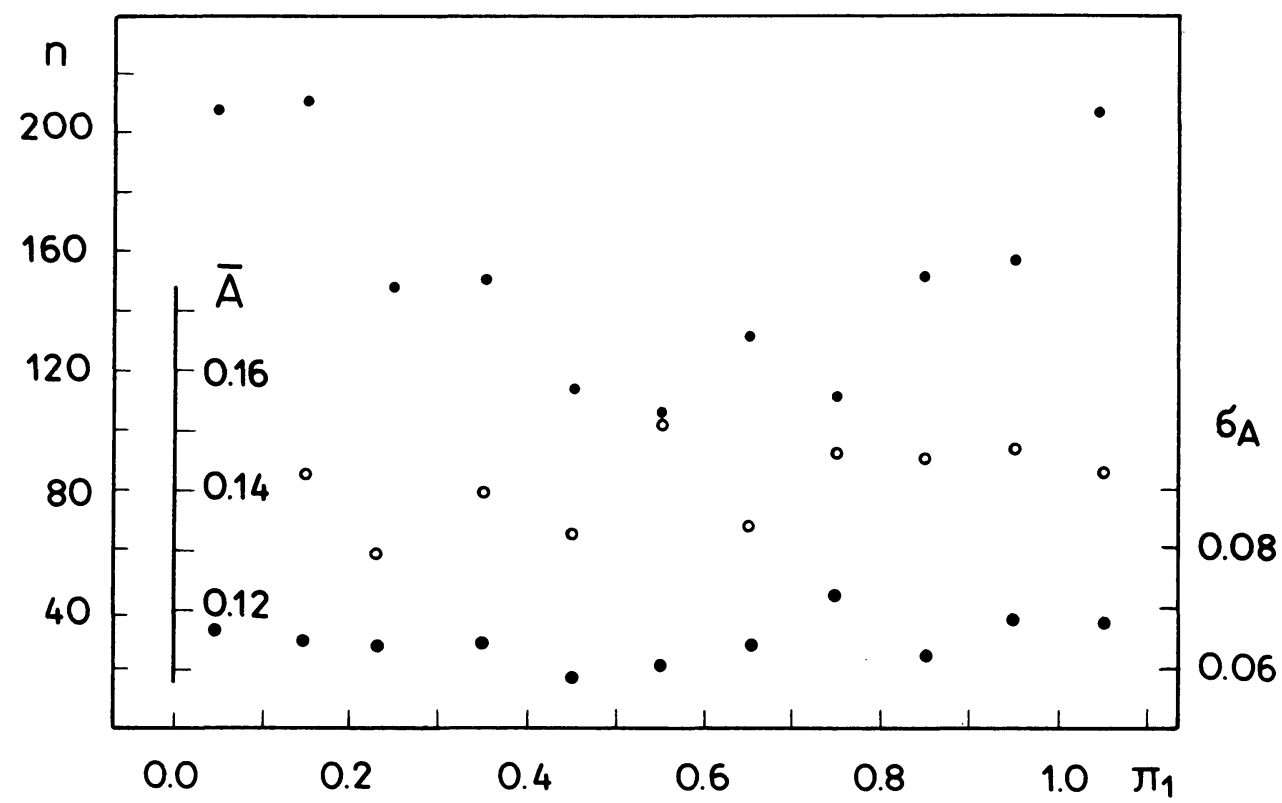

Fig. 2. The distribution function $n\left(\Pi_{1}\right)$ (dots), the mean value of the proper eccentricity, $A$ (open circles), and the dispersion of $A$ (black circles) vs the proper longitude of the perihelion, $\Pi_{1}$, are shown.

similar. (3) However, the relations $\bar{\varphi}(\Pi)$ and $A\left(\Pi_{1}\right)$ are not similar, as $\bar{\varphi}(\Pi)$ has been disturbed by the secular perturbations due to the major planets.

More interesting results are derived for the semimajor axis, $a$, the inclination, $i$, and the proper inclination, $B$. The relations, $a\left(\sigma_{i}\right)$ and $a\left(\sigma_{B}\right)$, dispersions $\sigma_{i}$ and $\sigma_{B}$ as functions of $a$, which are shown in Figure 3, do not lie on straight lines, and their deviations from them may be explained by Jupiter's large action due to commensurability.

Figure 4 shows similar relations, $a(\bar{i})$ and $a(\bar{B})$, the mean values of the inclination and of the proper inclination vs the semimajor axis.

By assuming that the relations $a\left(\sigma_{i}\right), a\left(\sigma_{B}\right), a(\bar{i})$ and $a(\bar{B})$ are linear with respect to $a$, the following expressions are derived by the method of least squares:

$$
\begin{array}{rlrl}
a & =0.221 \sigma_{i}+1.82, & a & =10.81 \sigma_{B}+1.87 \\
\pm 0.027 \pm 0.12 & \pm 0.28 \pm 0.08 \\
a=0.222 \bar{i}+0.90, & a & =7.22 \bar{B}+1.63 . \\
\pm 0.029 \pm 0.09 & \pm 0.22 \pm 0.09
\end{array}
$$




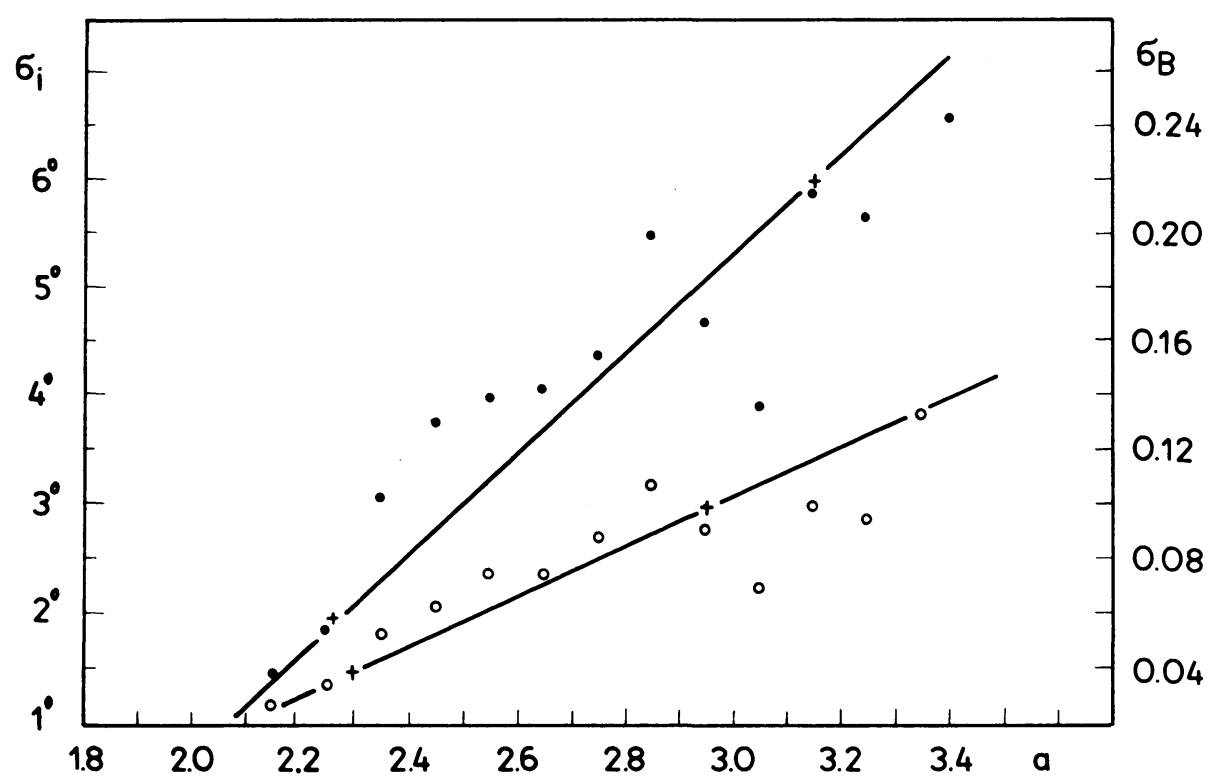

Fig. 3. The dispersion of the inclination, $i$ (dots), and that of the proper inclination, $B$ (open circles), vs the semimajor axis, $a$, are shown.

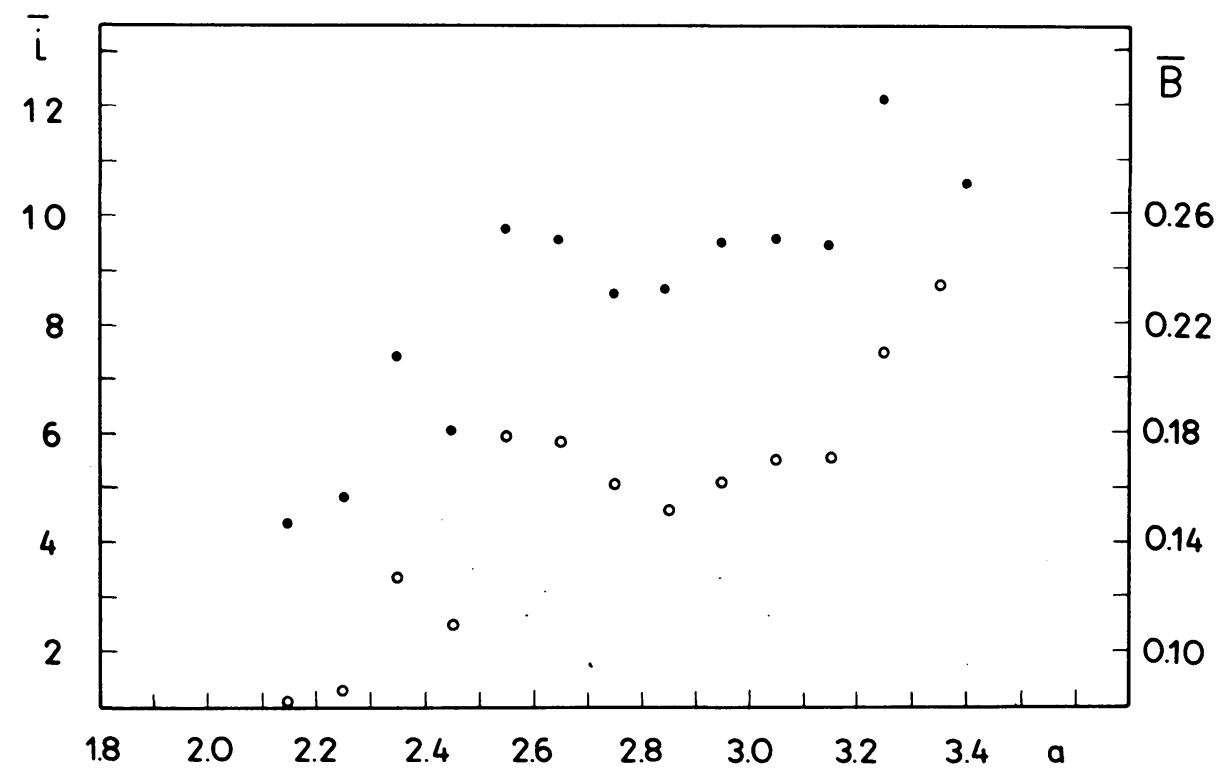

Fig. 4. The mean value of the inclination, $i$ (dots), and that of the proper inclination, $B$ (open circles), vs the semimajor axis, $a$, are shown. 
For the relations $a\left(\sigma_{i}\right)$ and $a\left(\sigma_{B}\right)$ the values of $a_{0}$, the values of $a$, for which the dispersions vanish, are almost identical. This will suggest that the relation of type $D\left(\sigma_{C}\right)$ is more stable than that of $D(\bar{C})$, where $C$ and $D$ are arbitrary orbital elements.

By similar ways the relations $Q\left(\sigma_{i}\right), Q\left(\sigma_{B}\right), H\left(\sigma_{A}\right)$ and $H\left(\sigma_{B}\right)$, where $Q=a(1+e)$, and $H$ is Jacobi's constant, are derived. Of these $H\left(\sigma_{A}\right)$ and $H\left(\sigma_{B}\right)$ cannot be represented by straight lines well, as near $H=920$ the relations are disturbed by Hill's instability.

By assuming that the asteroid ring was originated due to disintegration of a large planet, that the relations of type $D\left(\sigma_{C}\right)$ are invariable and that the original value of any orbital element is derived by putting $\sigma_{C}=0$ in the relation, the following set of values are determined as the orbital elements of the mother planet of asteroids:

$$
\begin{array}{rrrr}
a_{0}=1.85, & Q_{0}=2.24, & H_{0}=1109, & e_{0}=0.196 . \\
\pm 0.19 & \pm 0.10 & \pm 9 & \pm 0.043
\end{array}
$$

These values are not so different from those for Mars, for which $H_{M}=1278$, while the mean value of $H$ for all the asteroids is 943 . Therefore, we may conclude that the mother planet of the asteroid ring was Mars.

Similar computations are made for the Perseid meteor stream, which is known to have originated from the Comet 1862 III. The results are:

$$
a_{0}=24.2 \mathrm{AU}, \quad q_{0}=0.979 \mathrm{AU}, \quad e_{0}=0.960 .
$$

On the other hand they are $a=24.29, q=0.972$ and $e=0.960$ for the Comet 1862 III. The agreement is quite satisfactory (Gąska, 1973).

\section{References}

Brouwer, D.: 1950, Astron. J. 56, 9-32.

Gąska, S. : 1970, Bull. Toruń 45, 1.

Gąska, S.: 1973, Bull. Toruń (in press).

Sultanov, G. F. and Ibragimov, N. B.: 1962, Katalog postojannytr Jacobi, Baku. 